ARTIGO ORIGINAL

ISSN 1677-5090

(C) 2018 Revista de Ciências Médicas e Biológicas

DOI: http://dx.doi.org/10.9771/cmbio.v17i3.28629

\title{
Descrição da frequência de variantes genéticas no gene da endoglina em uma população do Nordeste do Brasil
}

\author{
Description of the frequency of genetic variants in the endoglin gene in a population of \\ Northeast Brazil
}

Yasmim Cristina Ferreira de Almeida ${ }^{1}$, Keina Maciele Campos Dourado ${ }^{2}$, Camila Alexandrina Figueiredo ${ }^{3 *}$

${ }^{1}$ Mestranda do Programa de Pós-Graduação de Processos Interativos de Órgãos e Sistemas, UFBA; ${ }^{2}$ Pós-Doutoranda em Farmácia, Instituto de Ciências da Saúde, UFBA; ${ }^{3}$ Professora Associada II , Instituto de Ciências da Saúde, UFBA.

\begin{abstract}
Resumo
Introdução: a endoglina (ENG, CD105) é um co-receptor da família transforming growth factor-beta e participa da regulação de processos celulares como proliferação, diferenciação, migração e apoptose. ENG é mais conhecida por sua expressão em células endoteliais, desempenhando papel importante na angiogênese e vasculogênese, porém sua expressão já foi associada a diferentes desfechos patogênicos, inclusive devido a mutações no gene ENG. Objetivos: descrever a frequência de variantes genéticas no gene ENG, comparar com populações ancestrais e analisar as variantes genéticas que possam estar envolvidas em processos patogênicos em outras populações. Metodologia: foi utilizado o banco de dados do programa SCAALA (Social Change Asthma and Allergy in Latin America) para a população do estudo, sendo genotipado 1309 indivíduos usando o chip Illumina 2.5 Human Omni Bead e feitas análises in silico utilizando plataformas on-line. Resultados: as variantes genéticas rs10987746, rs10121110, rs11792480 e rs16930129 apresentaram frequência de menor alelo entre 16 a $48 \%$ na população estudada, as quais foram mais reiteradamente próximas do padrão africano que do europeu. Os SNVs foram relacionados aos mecanismos regulatórios genéticos conhecidos, pressupondo que essas variantes não estejam envolvidas diretamente em processos funcionais. Conclusão: são necessárias maiores investigações referentes aos mecanismos funcionais deste gene, visto que a endoglina participa de uma gama de processos celulares importantes e mais esforços devem ser feitos para estudos genéticos na população brasileira, considerando a mistura de populações.

Palavras-Chave: Endoglina. Antígeno CD105. Polimorfismo de Nucleotídeo Único
\end{abstract}

\begin{abstract}
Introduction: the endoglin (ENG, CD105) is a coreceptor of the family transforming growth factor-beta and participates in the regulation of cellular processes such as proliferation, differentiation, migration and apoptosis. ENG She is best known for your expression in endothelial cells, playing an important role in angiogenesis and vasculogenesis, but its expression has already been associated with different pathogenic outcomes, including due to mutations in the ENG gene. Objectives: describe the frequency of genetic variants in the ENG gene in the population of northeastern Brazil, compare with ancestral populations and analyze genetic variants that may be involved in pathogenic processes in other populations. Methodology: we used the SCAALA program database (Social Change Asthma and Allergy in Latin America) for the population of the study, and the DNA of 1309 individuals were genotyped using the Illumina chip 2.5 Human Omni Bead and made in silico analysis. Results: the SNVs rs10987746, rs10121110, rs11792480 and rs16930129 presented lower allele frequency between 16 to $48 \%$ in the population studied, which were more consistently next African European standard. The SNVs were related to known genetic regulatory mechanisms assuming that these variants are not directly involved in functional processes. Conclusion: further investigation regarding the functional mechanisms of this gene are necessary, since the endoglin participates in a range of important cellular processes and more efforts should be made for genetic studies in the Brazilian population, considering the mixture of populations.
\end{abstract}

Keywords: Endoglin. CD105 Antigen. Single Nucleotide Polymorphism

\section{INTRODUÇÃO}

A endoglina, também conhecida como ENG e CD105, é uma glicoproteína transmembranar que funciona como um receptor auxiliar da superfamília do transforming growth factor-beta (TGF- $\beta$ ) (GOUGOS; LETARTE, 1990), mais conhecida por sua expressão abundante em células endoteliais ativas, desempenhando um papel crucial na angiogênese (LEBRIN et al., 2004) e no desenvolvimento

Correspondente/Corresponding: *Camila Alexandrina Figueiredo - Instituto de Ciências da Saúde, Universidade Federal da Bahia - Av. Reitor Miguel Calmon S/N, Vale do Canela, Salvador-Ba, Cep: 40110-100. - Tel/ Fax: +55 (71) 3283-892. - E-mail: cavfigueiredo@gmail.com vascular (BOURDEAU; DUMONT; LETARTE, 1999). A endoglina é um receptor do tipo III da família TGF- $\beta$ e atua em interação com as isoformas TGF- $\beta 1$ e TGF- $\beta 3$, através da associação a receptores tipo I como ALK1 e ALK5 e tipo II como TGFßR-II, iniciando vias de sinalização celulares mediadas através da fosforilação de proteínas SMADs no citoplasma, regulando processos celulares de proliferação, diferenciação, migração (LEBRIN et al., 2004) e apoptose (LI, 2003).

O gene ENG humano fica localizado no cromossomo 9q34.11 (FERNÁNDEZ-RUIZ et al., 1993), codifica 15 diferentes éxons e sua transcrição é conduzida por uma região promotora contendo duas regiões ricas em caixa 
GC (sequência consenso GGGCGG) permitindo a ligação a fatores de transcrição, como Sp1 (RÍUS et al., 1998). Mutações em região não codificadora, como na região promotora proximal, já foram descritas. Nesses casos, a regulação transcricional é afetada e impede a ligação de fatores de transcrição, como Sp1, tendo como desfecho um fenótipo patogênico, como a telangiectasia hemorrágica hereditária tipo 1 (HHT1) (ALBIÑANA et al., 2017).

A expressão de endoglina já foi associada a alguns desfechos: como já mencionado, a telangiectasia hemorrágica hereditária tipo 1 (HHT1), também conhecida como Síndrome de Rendu-Osler-Weber, devido a mutações no gene $E N G$, já sendo bastante estudado neste contexto (SHOVLIN, 2010); pré-eclâmpsia associada a níveis aumentados de ENG solúvel (sENG, sCD105) (VENKATESHA et al., 2006); tumores sólidos de localizações diversas (KASSOUF et al., 2004; PARDALI et al., 2011; SAAD et al., 2003, 2004); metástases associadas a nível elevado de sENG (TAKAHASHI et al., 2001); mieloma múltiplo (PRUNERI et al., 2002); e mais recentemente a leucemias agudas (DOURADO et al., 2017).

Considerando que a população brasileira seja miscigenada, tri-híbrida - descendente de africanos, europeus e ameríndios - é importante descrever a frequência das variantes genéticas em população do Nordeste brasileiro, comparando com população ancestral de maioria africana e europeia (LIMA-COSTA et al., 2015) e analisar as variantes genéticas em ENG que podem estar envolvidas em processos patogênicos já descritos anteriormente em outras populações.

\section{METODOLOGIA}

\section{População do Estudo}

Foi utilizado o banco de dados do Programa Social Change, Asthma and Allergy in Latin America (SCAALA) da cidade de Salvador, localizada no Nordeste do Brasil com uma população de 2,9 milhões de pessoas. $O$ desenho do estudo já foi relatado em outros trabalhos (BARRETO et al., 2006; FIGUEIREDO et al., 2011) e a população do estudo compreendeu 1309 crianças entre 4 e 11 anos, sem relação sanguínea. O estudo foi aprovado pelo Comitê de Ética em Pesquisa do Instituto de Saúde Pública (CEP-ISC, registro 003-05) da Universidade Federal da Bahia e pelo Conselho Nacional de Ética em Pesquisa (CONEP, número de resolução 15 895/2011). O termo de consentimento livre e esclarecido foi obtido do responsável legal ou dos pais de cada criança.

\section{Extração de DNA e Genotipagem}

A extração do DNA foi realizada a partir de amostras de sangue de acordo com o protocolo do kit Quiagen (Gentra Puregene Blood Kit; Hilden, Alemanha). Todas as amostras dos 1309 indivíduos foram genotipadas utilizando o Illumina BeadChip HumanOmni 2.5, uma plataforma de varredura ampla do genoma com 2,5 milhões de marcadores que foram genotipados através do Consórcio EPIGEN-Brasil (https://epigen.grude.ufmg.br). Para este estudo, a informação genética foi extraída das posições 127815012 a 127854773 , localizada no cromossomo 9 (9q34.11 - localização citogenética).

\section{Análises In silico}

Análises in silico foram conduzidas para variação de nucleotídeo único (em inglês: single nucleotide variation $S N V$ ) selecionados através da frequência dessas variantes no gene $E N G$, utilizando o filtro para frequência de menor alelo (em inglês: minor allele frequency $-M A F$ ) através do software PLINK 1.90 beta (PURCELL et al., 2007). A função de cada variação genética foi obtida através do National Center for Biotechnology Information (NCBI) (www.ncbi. nlm.nih.gov).

O banco de dados RegulomeDB (www.regulomedb. org) foi utilizado para a interpretação de funções regulatórias de regiões intergênicas no genoma humano. Este banco utiliza um conjunto de dados do ENCODE (enciclopédia de elementos de DNA) e outras fontes. RegulomeDB possibilita identificar um suposto potencial regulatório e variantes funcionais através de um sistema de pontuação (score), variando de 1 a 6 , em que pontuações mais baixas estão associadas ao aumento da evidência de que a variante esteja localizada em uma região funcional, desta forma, podendo apresentar consequência funcional (BOYLE et al., 2012).

O rSNPBase (http://rsnp.psych.ac.cn) é uma baco de dados que permite a obtenção de informações quanto a elementos regulatórios dos SNVs que foram previamente apoiados experimentalmente. As vias de regulação envolvem região proximal, distal e regulação pós transcricional através de miRNA (incluindo miRNAs maduros) e proteína de ligação a RNA (região identificada por imunoprecipitação de RNA), possibilitando a identificação dos possíveis genes regulados.

Para analisar e comparar a frequência de alelos nas populações ancestrais foi utilizada a plataforma de dados do International Genome Sample Resource (IGSR) (www. internationalgenome.org) através de dados do Ensembl, um navegador de genomas, utilizando a referência de genoma anterior GRCh37p.13.

\section{RESULTADOS}

A Tabela 1 apresenta os detalhes para as variantes genéticas examinadas em ENG. Os 4 SNVs analisados foram selecionados de acordo com a presença de resultados de outros estudos que associaram os SNVs a desfechos patogênicos. Os SNVs apresentaram MAF superior a 16\% na população estudada, sendo variantes intrônicas: rs10987746 com MAF de 48\% para alelo T, rs10121110 com $44 \%$ para alelo A, rs $11792480 \mathrm{com} 16 \%$ para alelo $A$ e variante exônica: rs16930129 com MAF de 21\% para o alelo T, na população estudada. 
Tabela 1 - Pares de base, alelos, frequência de menor alelo e função das variantes genéticas de ENG.

\begin{tabular}{llllll}
\hline $\mathrm{CHR}^{\mathrm{a}}$ & Variante & $\mathrm{A} 1^{\mathrm{b}}$ & $\mathrm{A} 2^{\mathrm{c}}$ & $\mathrm{MAF}$ & Função \\
\hline 9 & rs10987746 & $\mathrm{T}$ & $\mathrm{C}$ & 0.48 & intron variant \\
9 & rs10121110 & $\mathrm{A}$ & $\mathrm{G}$ & 0.44 & intron variant \\
9 & rs11792480 & A & G & 0.16 & intron variant \\
9 & rs16930129 & T & C & 0.21 & synonymous codon, utr variant 5 prime \\
\hline
\end{tabular}

Fonte: Dados do estudo.

Nota: ${ }^{\mathrm{a}}$ cromossomo ${ }^{\mathrm{b}}$ menor alelo, calelo ancestral.

Os SNVs foram relacionados aos mecanismos regulatórios conhecidos e identificados como "sim" ou "não" na Tabela 2. Os rs10987746, rs11792480 e rs16930129 estão envolvidos em regulação proximal. Todos os SNVs descritos podem estar envolvidos em regulação distal e em regulação mediada por proteína de ligação a RNA. Além disso, a mesma tabela apresenta dados relacionados ao RegulomeDB, indicando as características regulató- rias e funcionais através do score de cada variante: os rs10987746, rs10121110 e rs11792480 apresentaram scores de 4, 5 e 4, respectivamente. Esses valores pressupõe que essas variantes não estejam envolvidas diretamente em processos funcionais, pois há evidências mínimas de ligação. Já o rs16930129, única variante exônica, não possui score no RegulomeDB.

Tabela 2 - Dados referentes à função dos SNVs em ENG a partir de análises in silico, utilizando rSNPBase e RegulomeDB.

\begin{tabular}{|c|c|c|c|c|c|}
\hline SNV & Regulação proximal & Regulação distal & Regulação miRNA & $\begin{array}{c}\text { Regulação mediada por proteína } \\
\text { de ligação a RNA }\end{array}$ & RegulomeDB Score $^{b}$ \\
\hline rs10987746 & $\operatorname{sim}$ & $\operatorname{sim}$ & não & $\operatorname{sim}$ & 4 \\
\hline rs10121110 & não & $\operatorname{sim}$ & não & $\operatorname{sim}$ & 5 \\
\hline rs11792480 & $\operatorname{sim}$ & $\operatorname{sim}$ & não & $\operatorname{sim}$ & 4 \\
\hline rs16930129 & $\operatorname{sim}$ & $\operatorname{sim}$ & não & $\operatorname{sim}$ & - \\
\hline
\end{tabular}

Fonte: Dados do estudo.

Nota: ${ }^{a}$ Anotação de RegulomeDB score.

As frequências alélicas da população brasileira (BRA) e de seus ancestrais, africanos (AFR) e europeus (EUR) são apresentadas na Figura 1. O rs10987746 apresenta alelo C como referência e frequência de $52 \%$ na população brasileira, $46 \%$ em africanos e $45 \%$ em europeus, sendo T menor alelo. O rs16930129 apresenta alelo C como referência e frequências de $79 \%, 76 \%$ e $90 \%$, respecti- vamente, sendo T menor alelo. O rs10121110 apresenta alelo $\mathrm{G}$ como referência e frequências de $56 \%, 66 \%$ e $40 \%$, respectivamente, sendo $A$ menor alelo, porém na população europeia o alelo $A$ é mais frequente com $60 \%$. O rs 11792480 apresenta alelo $\mathrm{G}$ como referência e frequências de $84 \%, 97 \%$ e $66 \%$, respectivamente, sendo A menor alelo. 
Figura 1 - Frequências alélicas dos SNVs analisados em ENG em população brasileira, africana e europeia
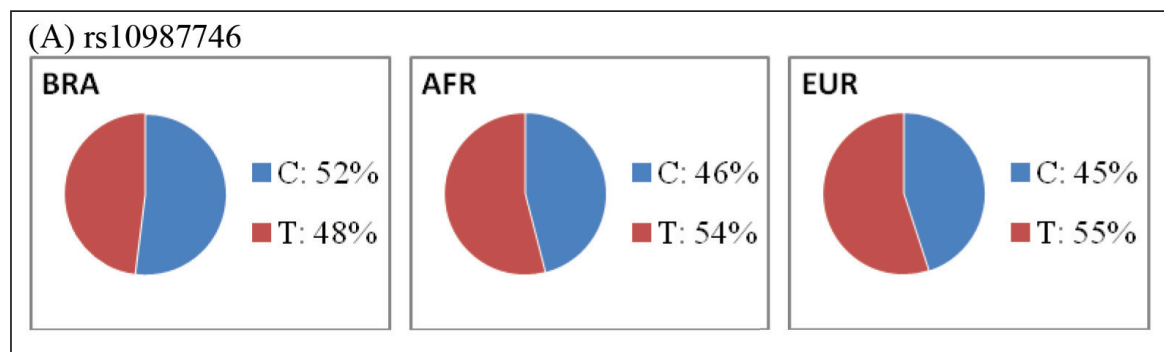

Legenda:

(B) rs 16930129
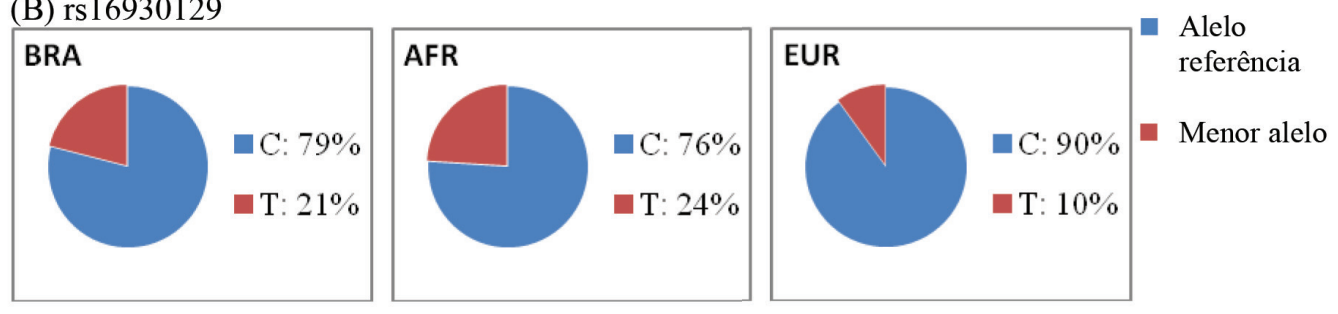

(C) rs 10121110
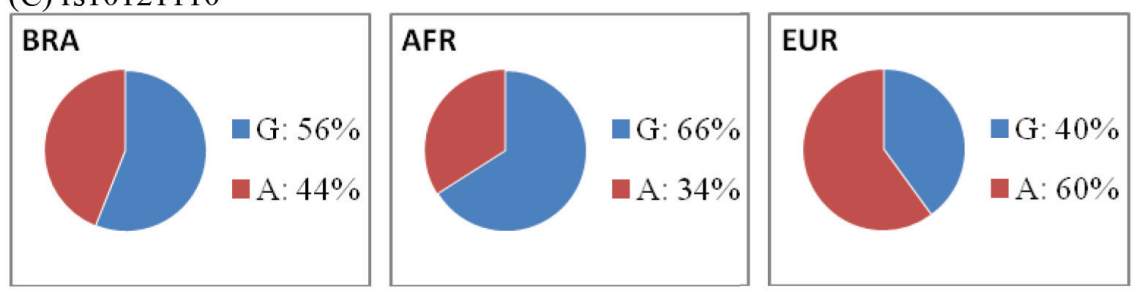

(D) rs 11792480
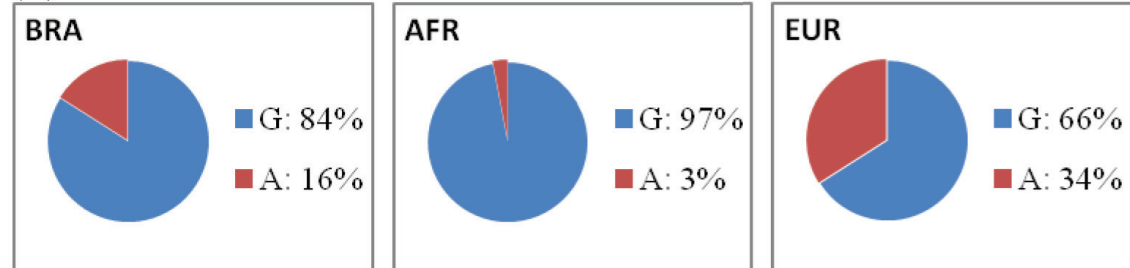

Fonte: Dados do estudo para frequências alélicas da população brasileira e Plataforma IGSR para frequências alélicas das populações africana e europeia.

\section{DISCUSSÃO}

Este estudo teve como objetivo descrever a frequência de variantes genéticas no gene ENG em uma população do Nordeste do Brasil, comparando com populações ancestrais, com o objetivo de conhecer as variantes que podem estar envolvidas em processos patogênicos já descritos anteriormente em outras populações. As diferentes variantes foram mais reiteradamente próximas do padrão africano que do europeu (Figura 1). Segundo dados do EPIGEN, a ancestralidade africana é mais predominante na população de Salvador, com média de $50,5 \%$, enquanto que europeus tem ancestralidade média de $42,4 \%$ e por último os americanos nativos, com média de 5,8\% (LIMA-COSTA et al., 2015).

Um estudo realizado nas Antilhas Holandesas, composta por uma população miscigenada, mostrou que entre Holandeses de origem caucasiana (mais predominante) e africana, o rs10987746 teve como alelo de risco o C-selvagem associado fracamente, porém, significativamente, a condição genética de malformação arteriovenosa (MAV) pulmonar em portadores de HHT1 [risco relativo $=1,3$ $(1,0018-1,7424)]$, alelo herdado de pais não afetados. 0 alelo C ocorreu em 56,5\% dos pacientes HHT1 com MAV pulmonar e $43,5 \%$ entre os HHT1 sem MAV pulmonar. Em modelo dominante, o mesmo alelo foi associado a baixos níveis de expressão de ENG $(P=0.004)$ em linhagens celulares linfoblastóides humanas (LETTEBOER et al., 2015). Este fator pode ser explicado devido ao envolvimento do rs10987746 em mecanismos reguladores (Tabela 2), podendo desempenhar papel importante na regulação transcricional de ENG.

A HHT1 é uma desordem autossômica dominante em consequência de mutação com perda de função predominantemente em ENG (SHOVLIN; LETARTE, 1999), carac- 
terizada pela formação defeituosa de vasos sanguíneos, ocorrendo recorrentes hemorragias por lesões vasculares e possui entre as consequências graves a ocorrência de MAV pulmonar (MCALLISTER et al., 1994). O alelo-C selvagem do rs10987746 apresentou uma associação fraca, porém significativa, com o risco aumentado de MAV pulmonar em pacientes com HTT1, sendo esta relação mais associada quando em homozigose. Na nossa população, o alelo C-rs 10987746 teve $52 \%$ de frequência (Figura 1).

Já em um estudo de um grupo da Universidade da Califórnia, São Francisco, pacientes autorreferidos brancos com MAV cerebral esporádica, ou seja, fora do contexto de HHT ou outra patologia cerebrovascular associada, apresentaram mecanismos patológicos comuns com o HHT ao apresentar variantes genéticas em ENG. O SNV rs10987746 apresentou uma associação marginal ( $P$ $=0.09)$ para MAV cerebral esporádica, assim como o rs16930129 ( $P=0.002)$ (PAWLIKOWSKA et al., 2005). A MAV cerebral ocorre em pacientes com HHT (MCALLISTER et al., 1994), sendo rara a ocorrência familiar de MAV cerebral esporádica (KAMIRYO et al., 2000).

O rs10987746 fica localizado no íntron 12, próximo a região final 3' do gene, desta forma, podendo estar associado com diferenças quantitativas, como em níveis de transcrição, ou qualitativas, como splicing diferencial, na proteína ENG (LETTEBOER et al., 2015), pois altera um sítio de ligação CCAAT, modulando potencialmente a transcrição (PAWLIKOWSKA et al., 2005). Já o rs16930129 é uma variante exônica que tem fenótipo silencioso reduzindo fator de splicing para SRp40 (PAWLIKOWSKA et al., 2005)

Os SNVs rs 11792480 e rs 10121110 foram associados à pré-eclâmpsia em mulheres brancas Norte-Americanas. 0 alelo G-rs 11792480 ocorreu em $71,7 \%$ das mulheres com pré-eclâmpsia e $63 \%$ em controles e o alelo A-rs 10121110 ocorreu em $66 \%$ e $55,3 \%$, respectivamente. Mulheres que herdaram o alelo A-rs10121110 em homozigose tiveram um risco aumentado em 2.29 vezes para desenvolver pré-eclâmpsia quando comparadas a mulheres com o genótipo $\mathrm{GG}(\mathrm{P}=0.008$, [99\% $\mathrm{Cl}: 1,02$ a 5,13]) (BELL et al., 2013).

No nosso estudo, o alelo G-rs11792480 teve a frequência de $84 \%$ e o alelo A-rs 10121110 de 44\% (Figura 1).

Os rs11792480 e rs10121110 estão envolvidos com mecanismos regulatórios e de transcrição (Tabela 3) e se localizam em uma região intrônica entre o segundo e terceiro éxons, fazendo parte da região gênica que inclui o promotor ENG (BELL et al., 2013), sendo assim, essas variantes podem implicar na ligação de fatores de transcrição como Sp1 e elementos SMADs (BOTELLA et al., 2001), desta forma, tendo impacto na transcrição e tradução do gene (BELL et al., 2013).

\section{CONCLUSÕES}

Até onde sabemos, este é o primeiro estudo que analisou, em um contexto genérico, a frequência de variantes genéticas de $E N G$ em uma população do Nordeste brasi- leiro (Salvador, Bahia). Desfechos importantes como MAV pulmonar em portadores de HHT1, MAV cerebral esporádica e pre-eclâmpsia foram associados em outros estudos com os alelos mais frequentes de SNVs no gene ENG, os quais também são mais frequentes em nossa população, exceto o alelo A-rs10121110 em relação a pré-eclâmpsia, o qual teve uma frequência menor na nossa população (44\%). As variantes genéticas analisadas participam de processos regulatórios, podendo impactar na transcrição e tradução da endoglina. Cabe ressaltar que os estudos referenciados tiveram brancos como a maioria da população, o que limita uma comparação fidedigna com os nossos achados, visto que as frequências da população do presente estudo sejam mais próximas das frequências de ancestrais africanos e o fator sexo não fora considerado. Mais investigações referentes aos mecanismos funcionais deste gene são necessárias, visto que participa de uma gama de processos celulares importantes e mais esforços devem ser feitos para estudos genéticos na população brasileira, considerando a mistura de populações.

\section{REFERÊNCIAS}

ALBIÑANA, V. et al. Mutation affecting the proximal promoter of Endoglin as the origin of hereditary hemorrhagic telangiectasia type 1. BMC med. genet., London, v. 18, n. 1, p. 1-9, 2017.

BARRETO, M. L. et al. Risk factors and immunological pathways for asthma and other allergic diseases in children: background and methodology of a longitudinal study in a large urban center in Northeastern Brazil (Salvador-SCAALA study). BMC pulm. med., London, v. 6, n. 1, p. 15, 23 Dec. 2006.

BELL, M. J. et al. Variation in endoglin pathway genes is associated with preeclampsia : a case - control candidate gene association study. 2013.

BOTELLA, L. M. et al. Identification of a critical sp1 site within the endoglin promoter and its involvement in the transforming growth factor-? $s$ timulation*. J. biol. chem., Baltimore, v. 276, n. 37, p. 34486-34494, 2001.

BOURDEAU, A.; DUMONT, D. J.; LETARTE, M. A murine model of hereditary hemorrhagic telangiectasia. J. clin. invest., New York, v. 104, n. 10, p. 1343-1351, 15 Nov. 1999.

BOYLE, A. P. et al. Annotation of functional variation in personal genomes using RegulomeDB. Genome res., New York, v. 22, n. 9, p. 1790-1797, 2012.

DOURADO, K. M. C. et al. Endoglin: a novel target for therapeutic intervention in acute leukemias revealed in xenograft mouse models. Blood, [S.I], v. 129, n. 18, p. 2526-2536, 2017.

FERNÁNDEZ-RUIZ, E. et al. Assignment of the human endoglin gene (End) to 9q34 $\rightarrow$ qter. Cytogenet. genome res., New York, v. 64, n. 3-4, p. 204-207, 1993.

FIGUEIREDO, C. A. et al. Evidence for a modulatory effect of IL-10 on both Th1 and Th2 cytokine production: the role of the environment. Clin. immunol., Orlando, v. 139, n. 1, p. 57-64, Apr. 2011.

GOUGOS, A.; LETARTE, M. Communication primary structure of endoglin , an glycoprotein of. J. biol. chem., Baltimore, v. 265, n. 15, p. $8361-8364,1990$

KAMIRYO, T. et al. Familial arteriovenous malformations in siblings. Surg. neurol., Boston v. 53, n. 3, p. 255-259, Mar. 2000. 
KASSOUF, W. et al. Whole-mount prostate sections reveal differential endoglin expression in stromal, epithelial, and endothelial cells with the development of prostate cancer. Prostate cancer prostatic dis., London, v. 7, p. 105-110, 2004.

LEBRIN, F. et al. Endoglin promotes endothelial cell proliferation and TGF-beta/ALK1 signal transduction. EMBO j., Oxford, v. 23, n. 20, p. 4018-4028, 13 Oct. 2004.

LETTEBOER, T. G. W. et al. Genetic variation in the functional ENG allele inherited from the non-affected parent associates with presence of pulmonary arteriovenous malformations in hereditary hemorrhagic telangiectasia 1 (HHT1) and may influence expression of PTPN14. Front. genet., Switzerland, v. 5, p. 1-9, 2015.

LI, C. ET AL. CD105 prevents apoptosis in hypoxic endothelial cells. J. cell science, London, v. 116, n. 13, p. 2677-2685, 2003.

LIMA-COSTA, M. F. et al. Genomic ancestry and ethnoracial self-classification based on 5,871 community-dwelling Brazilians (The Epigen Initiative). Sci. rep., London, v. 5, p. 9812, 27 Apr. 2015.

MCALLISTER, K. A. et al. Endoglin, a TGF-beta binding protein of endothelial cells, is the gene for hereditary haemorrhagic telangiectasia type 1. Nat. genet., New York, v. 8, n. 4, p. 345-351, 1994.

PARDALI, E. et al. Critical role of endoglin in tumor cell plasticity of Ewing sarcoma and melanoma. Oncogene, Basingstoke, v. 30, p. 334-345, 2011.

PAWLIKOWSKA, L. et al. Polymorphisms in transforming growth factor-beta-related genes ALK1 and ENG are associated with sporadic brain arteriovenous malformations. Stroke, Dallas, v. 36, n. 10, p. 2278-2280, 1 Oct. 2005.

PRUNERI, G. et al. Microvessel density, a surrogate marker of angioge- nesis, is significantly related to survival in multiple myeloma patients. Br. j. haematol., Oxford, v. 118, n. 3, p. 817-820, 2002.

PURCELL, S. et al. PLINK: a tool set for whole-genome association and population-based linkage analyses. Am. J. Hum. Gen., Baltimore, v. 81, n. 3, p. 559-575, 2007.

RÍUS, C. et al. Cloning of the Promoter region of human endoglin, the target gene for hereditary hemorrhagic telangiectasia type 1. Blood, [S.I], v. 92, n. 12, p. 4677-4690, 1998.

SAAD, R. S. et al. Endoglin (CD105) expression in endometrial carcinoma. Int. j. gynecol pathol., New York, v. 22, n. 3, p. 248-253, 2003.

SAAD, R. S. et al. Endoglin (CD105) and vascular endothelial growth factor as prognostic markers in colorectal cancer. Mod. pathol., Baltimore, v. 17, n. 2, p. 197-203, 2004.

SHOVLIN, C. L. Hereditary haemorrhagic telangiectasia: pathophysiology, diagnosis and treatment. Blood rev., Edinburgh, v. 24, n. 6, p. 203-219, 2010

SHOVLIN, C. L.; LETARTE, M. Hereditary haemorrhagic telangiectasia and pulmonary arteriovenous malformations: issues in clinical management and review of pathogenic mechanisms. Thorax, London, v. 54, p. 714-729, 1999.

TAKAHASHI, N. et al. Association of serum endoglin with metastasis in patients with colorectal, breast, and other solid tumors, and suppressiveeffect of chemotherapy on the serum endoglin. Clin. cancer res., Philadelphia, v. 7, n. 3, p. 524-532, Mar. 2001.

VENKATESHA, S. et al. Soluble endoglin contributes to the pathogenesis of preeclampsia. Nat. med., New Yorkm v. 12, n. 6, p. 642-649, 4 June 2006.

Submetido em: $14 / 11 / 2018$

Aceito em: 29/11/2018 